\title{
Online Measurement and Control System Based on DeviceNet Fieldbus
}

\author{
Hu Zhou ${ }^{1,2, a}$, Jiang Liu ${ }^{2, b}$, Guangping Qi ${ }^{1,2, c}$, Zongyou Xiang ${ }^{1, d}$ \\ ${ }^{1}$ Beijing Aerospace Automatic Control Institute, Beijing, China \\ ${ }^{2}$ National Key Laboratory of Science and Technology on Aerospace Intelligence Control, Beijing, \\ China
}

aemail: zhouhu0728@sohu.com, bemail:syq_syq@sohu.com, email:184236006@qq.com, demail:277792823@qq.com

Keywords: DeviceNet; Fieldbus; welding; control system; automobile production line

\begin{abstract}
In order to ensure the quality of welding line in Dongfeng Honda Automobile Corporation, the online automatic measurement and control station is designed. The operational principle of the station is introduced firstly, then the architecture of the system including power supply and distribution unit, control unit, measurement and display unit is expounded, the process for information interaction between the PLC of the station and the production line is illuminated at last. It is proved by the field experiment results that the system is running reliably and the measurement quality of welded car body can be improved efficiently.
\end{abstract}

\section{Introduction}

DeviceNet fieldbus is the lower level of the optimal industrial control network technology by Rockwell Automation Co., Ltd in USA, which is a world class supplier for the solution of automatic control and information transport. Because the CAN bus communication controller chips are embedded into network nodes, industrial equipments such as motor drivers, limit switches, photoelectric sensors can be connneted into the bus control network directly. It is especially suitable for lower level control with high real-time performance in industrial field with DeviceNet fieldbus considering that cost is reduced by a wide margin compared with hard-wire and the communication ability among equipments is improved obviously with equipment level diagnostic function. DeviceNet has been the third part of the international standard IEC 62026, as well as the European standard EN 50325. As an open, cheap, reliable, efficient fieldbus, DeviceNet was adopted as national standard GB/T 18858.3-2002 in China. DeviceNet fieldbus has been supported by more and more manufacturers and there are millions of equipments with DeviceNet interface in world..

DeviceNet fieldbus is widely used in automatic control field. Taking automobile manufactory for example, DeviceNet fieldbus is applied to welding and assemble production line for AUDI A6, BORA A4, JETTA in FAW-Volkswagen Automobile Co. Ltd, welding production line in Dongfeng Honda Automobile Co. Ltd, welding and assemble production line in Zhangjiagang Mudan Automobile Factory, coating production line in Etsong(Qingdao) Industrial Co. Ltd, Bogie coating production line in Changchun Railway Vehicles Co. Ltd, welding, coating and assemble production line in Harbin Hafei Automobile Industrial Group Co. Ltd in China. The online automatic measurement and control station for welding quality control in Dongfeng Honda Automobile Co. Ltd is introduced in this paper..

\section{Design Object for the Measurement and Control Station}

Technical transformation is applied according to the layout of Dongfeng Honda Mobile Co. Ltd to the equipments and control system of the existing welding production line in order to meet the requirement of mixed welding production line in speed of 45 seconds per one car for three car types including CIVIC, SPIRIOR and CRV. The measurement and control station is designed to measure the key characters of the welded car body on line within a specified time in order to verify the quality of the car and avoid defect welded car body appeared in next process $[1,2]$. 


\section{Principle of the Measurement and Control Station}

The measurement and control station is located in the last of the welding production line. The integrated system of control and measurement consists of four industrial robots, four non-contact sensors, a programmable logic controller (PLC), a measurement computer, a data server, and a human-machine interaction unit (HMI), which are connected by four types: point to point hard wire, Ethernet based on TCP/IP protocol, coaxial cable for video information, DeviceNet fieldbus. The station specializes in interaction between the local PLC and the production line, measurement for the key characters of the car body, result display, data storage and remote query, which architecture is shown as Figure 1.

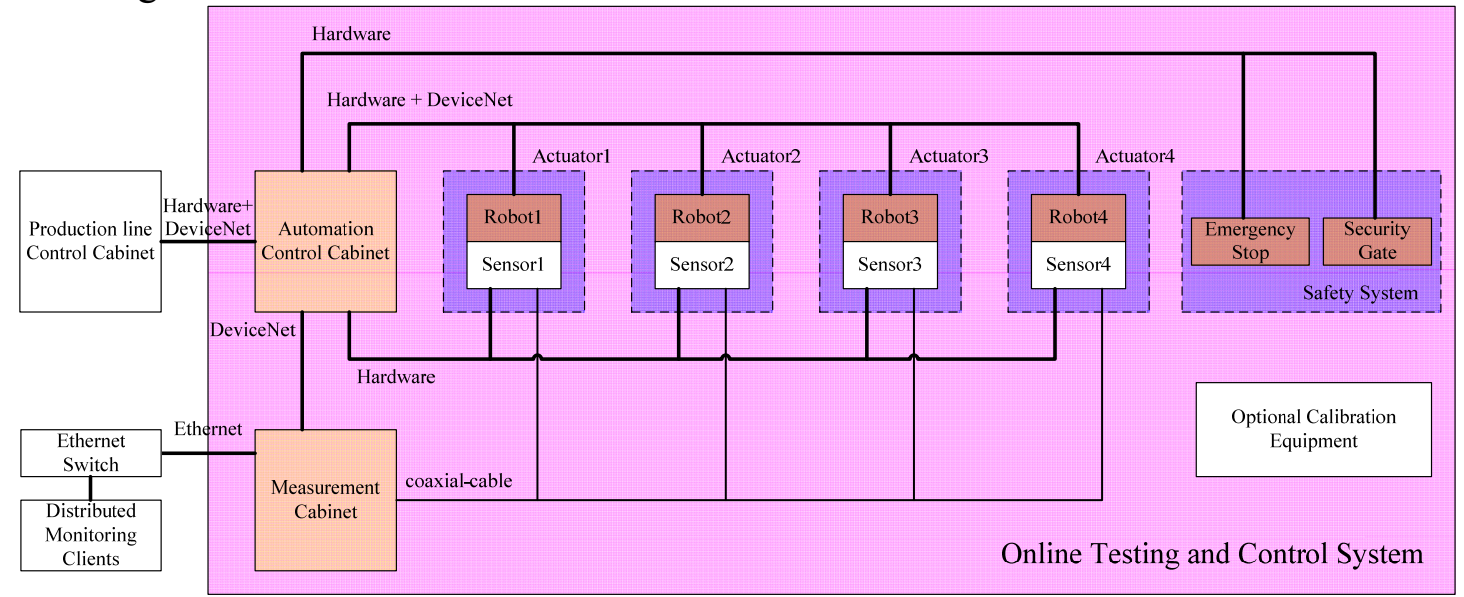

Fig.1. Architecture of the online measurement and control station

The welded car body is transported into the measurement and control station, and located stably and precisely by tools. The robots are controlled by the PLC to move with the sonsers in accordance with the predetermined trajectory according to the special car type subsequently, then the measurement results by sensors are sent to the measurement computer, by which we can determine whether the welded body is in the allowable limit range. The welded car body is sent to next workstation according to the quality conclusion [3,4], as shown in Figure 2.

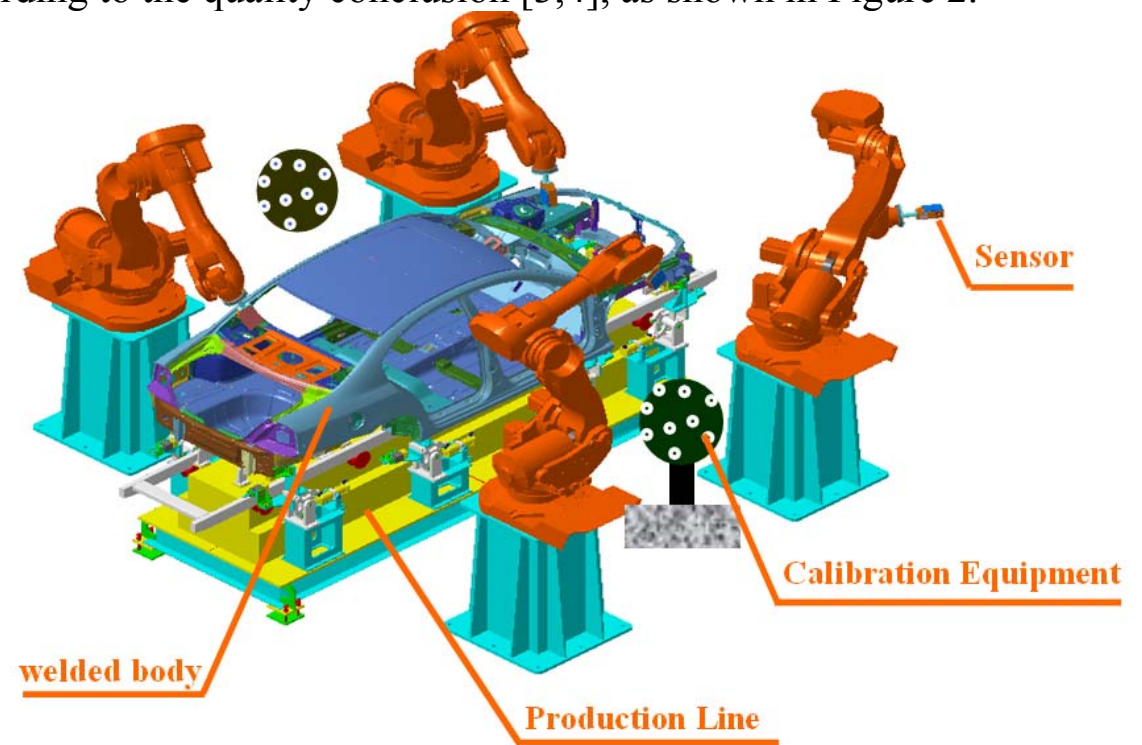

Fig.2. Architecture of the online measurement and control station

\section{Systematic Structure of the Measurement and Control Station}

Power supply and distribution unit. Two stage power supply and distribution schemes are applied in measurement and control station. The primary power scheme mainly involves heavy current devices of the station which input is from $220 \mathrm{~V}$ AC in the welding ship, whereas the secondary scheme mainly involves weak power supply in the station. Specific details about power supply and distribution is illuminate as follows: 
1) The input of $220 \mathrm{~V} \mathrm{AC}$ distributed by the primary scheme is converted for working voltage of the PLC with the power module OMRON PA204.

2) The power supplied for the HMI screen, control circuit, production line circuit, safety system, digital input and output module of the PLC is $24 \mathrm{~V}$ DC by quadratic transformation.

3) The power supplied for sensors is $12 \mathrm{~V}$ DC by quadratic transformation.

4) The measurement computer and data server can run continuously and placidly in any unexpected situations such as power off or power network fluctuation considering that uninterruptible power system (UPS) is installed in the measurement cabinet. The power input $220 \mathrm{~V}$ AC of the UPS is supplied by the output of the primary scheme.

5) Centralized power supply mode is adopted for all the robots in the welding ship, so there is no correlation with the power of the robots in the station.

Control Unit. Information between PLC and four robots is interacted mainly via DeviceNet fieldbus. Point to point, multiple masters, or master-slave communication mode is permitted, and there are 64 nodes at most for one DeviceNet network. Master-slave mode with baud rate of $125 \mathrm{Kbit} / \mathrm{s}$ is chose for the station, where the PLC is the master controller, whereas four robots and the measurement computer is slaves connected to the fieldbus. Topology of the control unit is shown in Figure 3.

A series of devices made by OMRON is used to build the fieldbus network. For example, CS1H-CPU64H module is used as CPU of the PLC, while CS1W-DRM21-V1 communication module is used as master node controller of the DeviceNet fieldbus. There are two $\mathrm{T}$ branch joints with their type OMRON DCN1-1C as the DeviceNet interface for the PLC. One of the branch joints and the PCI-DeviceNet interface conversion board in the measurement computer is connected by the special cable with the type OMRON DCA2-5C10, whereas another joint is connected to the robots'cabinets by the same type cable. Multi-branch joints with the type OMRON XW4B-05C4-TF-D are used in order to ensure reliability of the connection. There is no terminal resistance embedded in multi-branch joints comparing with $\mathrm{T}$ branch joint, so a special terminal resistance OMRON DRS1-T is required for the fieldbus network.

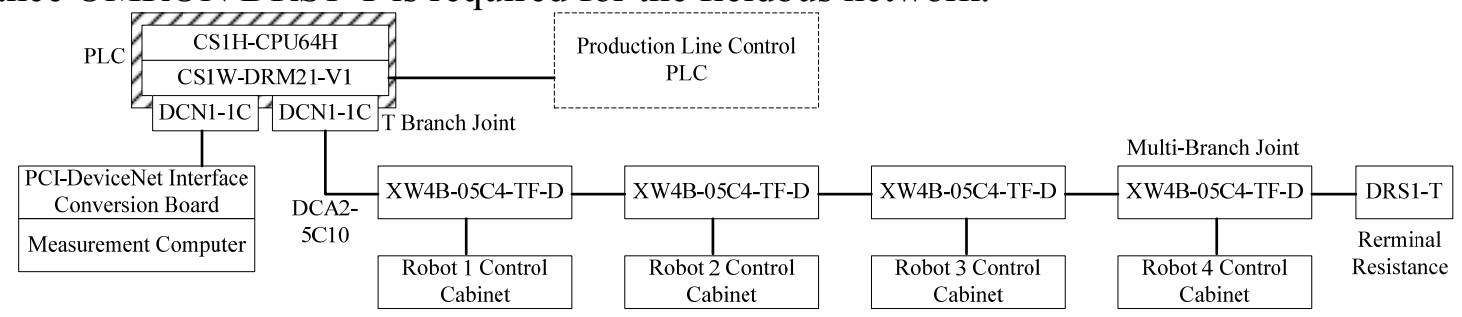

Fig.3. Topology of the fieldbus network

Control process with hard wire is better than DeviceNet in some situations such as application for safety, power driver (for example, the sensors power supply), and the special information interaction requirement standardized by the welding ship or determined by the devices themselves, shown as Figure 4. Hard wire is also used in part of the communication between production line and the digital input module OMRON ID261 or output module OMRON OD231/232 of the PLC, as well as part of the communication between robots and PLC $[5,6]$.

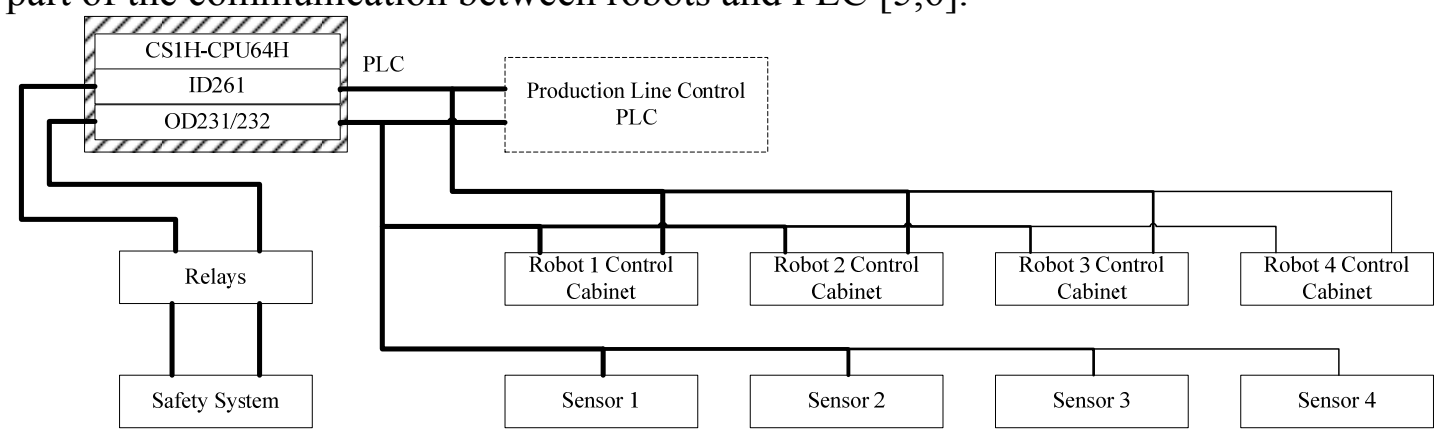

Fig.4. Topology of the hard wire

Measurement and Display Unit. A multi-channel video capture board made by Matrox is embedded in the measurement computer, while a camera and a laser is embedded in each sensor. Images of the welded car body shot by the cameras based on triangulation laser ranging principle 
are sent to the video capture board via four channel coaxial cable. After image process and data analysis, the measurement results are saved in the local computer and the data server for backup and query by the distributed clients located in the welding ship. Important results are also displayed in the touch screen of the HMI which data is received from PLC and measurement computer by serial ports. The topology of measurement and display unit is shown as Figure 5.

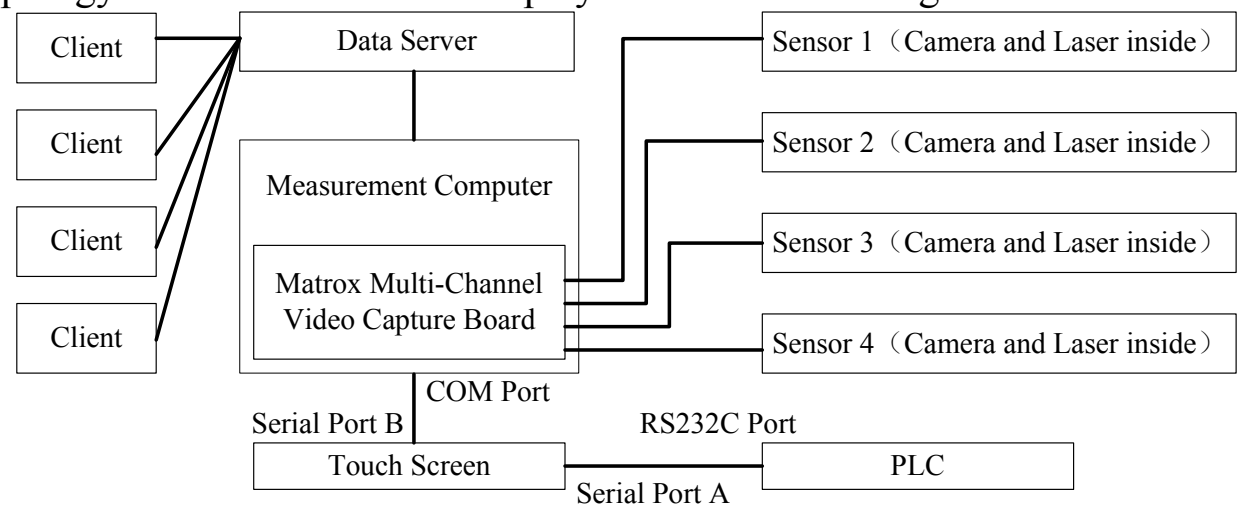

Fig.5. Topology of the measurement and display unit Production Line PLC

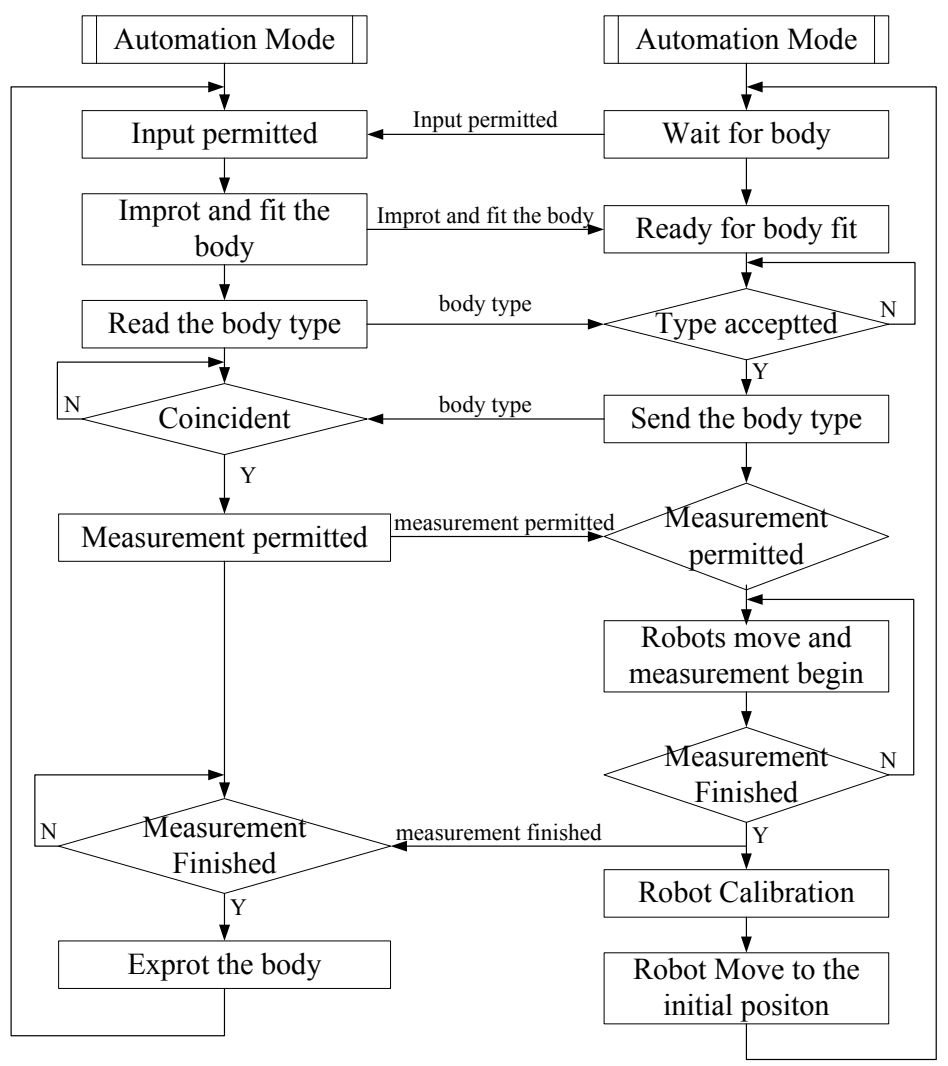

Fig.6. Signal interaction between local PLC and production line

Measurement and Control Process of the Station. The online measurement and control station based on PLC and DeviceNet fieldbus runs automatically in AUTOMATION mode, which means there is no emergency stop signal or security gate open signal sent from the safety system, all the robots locates in the initial positions, the measurement computer is $\mathrm{OK}$, and the status switch is set as "AUTO" position. Signal interaction between local PLC and production line can be illuminated briefly as Figure 6. If the measurement and control station is idle and waits for a new welded body, an "input permitted" signal is sent to the production line, then the body is transport to the station and fitted precisely by the production line. The type of the car body is read and sent to the PLC, and then the body type is returned verbatim. If the two body types sent to and received from the PLC are the same, a "measurement permitted" signal is sent to the PLC, then the robots move and measurement process begins; else retries body type validation process. If the measurement of the 
welded body is finished, the "measurement signal" is sent to the production line. Then the body is lifted and exported to the subsequent process while the robots move to the initial positions for next measurement cycle after calibration process.

\section{Conclusion}

Online measurement and control system based on DeviceNet fieldbus is designed in order to facilitate the communication and field control for the station. The reliability and efficiency of the station is proved by field running performance, which is met the requirement and expectation for Dongfeng Honda Automobile Corporation.

\section{References}

[1] J. G. Zhu, S. H. Ye, X. Y. Yang, et al. On-line Industrial 3D measurement techniques for large volume objects [J]. Key Engineering Materials, Vol. 295-296(2005), p.423-428.

[2] Hu. Zhou, Xuechao. Jiang, Yanqiu. Sun. Design on the real-time online automatic measurement system for body-in-white based on industrial robots [C]. 4th international conference on manufacturing science and engineering(ICMSE 2013), Vol. 694-697(2013), p.1684-1689.

[3] Motta. J. M., Garvalho. G. C., McMaster. R. S.. Robot calibration using a 3D vision-based measurement system with a single camera $[\mathrm{J}]$. Robotics and Computer Integrated Manufacturing, 2001, Vol. 17(6), 487-497.

[4] Georgis N, Petrou M, Kittler J. Error guided design of a 3D vision system [J]. IEEE transactions on pattern analysis and machine intelligence, 1998, 20(4), 366-379.

[5] Gatla C. S, Lumia R. Wood, J. Starr G. An automated method to calibrate industrial robots using a virtual closed kinematic chain [J]. IEEE transactions on robotics and automation, 2007, 11(23), 105-116.

[6] Acosta L. Marichal, G. N. Mereno, J. J. A robotic system based on neural network controllers [J]. Artifical intelligence in engineering, 1999, 13, 393-398. 